\title{
On Ramsey numbers of hedgehogs
}

\author{
Jacob Fox* and Ray $\mathrm{Li}^{\dagger}$
}

February 28, 2019

\begin{abstract}
The hedgehog $H_{t}$ is a 3 -uniform hypergraph on vertices $1, \ldots, t+\left(\begin{array}{l}t \\ 2\end{array}\right)$ such that, for any pair $(i, j)$ with $1 \leq i<j \leq t$, there exists a unique vertex $k>t$ such that $\{i, j, k\}$ is an edge. Conlon, Fox, and Rödl proved that the two-color Ramsey number of the hedgehog grows polynomially in the number of its vertices, while the four-color Ramsey number grows exponentially in the number of its vertices. They asked whether the two-color Ramsey number of the hedgehog $H_{t}$ is nearly linear in the number of its vertices. We answer this question affirmatively, proving that $r\left(H_{t}\right)=O\left(t^{2} \ln t\right)$.
\end{abstract}

\section{Introduction}

For a $k$-uniform hypergraph $H$, the Ramsey number $r(H)$ is the smallest $n$ such that any 2coloring of $K_{n}^{(k)}$, the complete $k$-uniform hypergraph on $n$ vertices, contains a monochromatic copy of $H$. Let $r(H ; q)$ denote the analogous Ramsey number for $q$-colorings, so that $r(H)=$ $r(H ; 2)$.

It is a major open problem to determine the growth of $r\left(K_{t}^{(3)}\right)$, the Ramsey number of the complete 3 -uniform hypergraph on $t$ vertices. It is known $[6,7]$ that there are constants $c, c^{\prime}>0$ such that

$$
2^{c t^{2}} \leq r\left(K_{t}^{(3)}\right) \leq 2^{2^{c^{\prime} t}}
$$

Erdős conjectured that $r\left(K_{t}^{(3)}\right)=2^{2^{\Theta(t)}}$, i.e. the upper bound is closer to the truth. Erdős and Hajnal gave some evidence that this conjecture is true by showing that $r_{3}\left(K_{t}^{(3)} ; 4\right) \geq 2^{2^{c t}}$, i.e. the four color Ramsey number of $K_{t}^{(3)}$ is double-exponential in $t$ (see, for example [9]).

Definition 1.1. The hedgehog $H_{t}$ is a 3-uniform hypergraph on $t+\left(\begin{array}{l}t \\ 2\end{array}\right)$ vertices $1, \ldots, t+\left(\begin{array}{l}t \\ 2\end{array}\right)$ such that, for each $1 \leq i<j \leq t$, there exists a unique vertex $k>t$ such that $\{i, j, k\}$ is an edge, and there are no additional edges.

*Department of Mathematics, Stanford University, Stanford, CA 94305. Email: jacobfox@stanford.edu. Research supported by a Packard Fellowship, and by NSF Career Award DMS-1352121.

${ }^{\dagger}$ Department of Computer Science, Stanford University, Stanford, CA 94305. Email: rayyli@cs.stanford.edu. Research supported by the National Science Foundation Graduate Research Fellowship Program under Grant No. DGE-1656518. 
We sometimes refer to the first $t$ vertices as the body of the hedgehog. For any $k \geq 4$, one can also define a $k$-uniform hedgehog $H_{t}^{(k)}$ on $t+\left(\begin{array}{c}t \\ k-1\end{array}\right)$, with a body of size $t$ and a unique hyperedge for every $k-1$-sized subset of the body. In this notation, we have $H_{t}=H_{t}^{(3)}$.

Hedgehogs are interesting because their 2-color Ramsey number $r\left(H_{t} ; 2\right)$ is polynomial in $t$, while their 4-color Ramsey number $r\left(H_{t} ; 4\right)$ is exponentially large in $t[10,5]$. This suggests that the bound $r\left(K_{t}^{(3)} ; 4\right) \geq 2^{2^{c t}}$ by Erdös and Hajnal may not be such strong evidence that $r\left(K_{t}^{(3)}\right)=2^{2^{\Theta(t)}}$.

Hedgehogs are also interesting because they are a natural family of hypergraphs with degeneracy 1. Degeneracy is a notion of sparseness for graphs and hypergraphs. For graphs, the degeneracy is defined as the minimum $d$ such that every subgraph induced by a set of vertices has a vertex of degree at most $d$. The Burr-Erdös conjecture [2] states that there exists a constant $c(d)$ depending only on $d$ such that the Ramsey number of any $d$ degenerate graph $G$ on $n$ vertices satisfies $r(G) \leq c(d) \cdot n$. Building on the work of Kostochka and Sudakov [11] and Fox and Sudakov [8], Lee [12] recently proved this conjecture. We can similarly define the degeneracy of a hypergraph as the minimum $d$ such that every subhypergraph induced by a subset of vertices has a vertex of degree at most $d$. Under this definition, Conlon, Fox, and Rödl [5] observe that the 4-uniform analogue of the Burr-Erdös conjecture is false: the 4 -uniform hedgehog $H_{t}^{(4)}$, which is 1-degenerate, satisfies $r\left(H_{t}^{(4)}\right) \geq$ $2^{c t}$. They also observe that the 3-uniform analogue of the Burr-Erdös conjecture is false for 3 or more colors: the 3 -uniform hedgehog, which is 1-degenerate, satisfies $r\left(H_{t} ; 3\right) \geq$ $\Omega\left(t^{3} / \log ^{6} t\right)$.

However, the analogue of the Burr-Erdős conjecture for 3-uniform hypergraphs and 2 colors remains open. In particular, it was not known whether the Ramsey number of the hedgehog $H_{t}$ is linear, or even near-linear, in the number of vertices, $t+\left(\begin{array}{l}t \\ 2\end{array}\right)$. Conlon, Fox and Rodl [5] show $r\left(H_{t} ; 2\right) \leq 4 t^{3}$, and, with the above in mind, ask if $r\left(H_{t} ; 2\right)=t^{2+o(1)}$. We answer this question affirmatively.

Theorem 1.2. If $t \geq 10$ and $n \geq 200 t^{2} \ln t+400 t^{2}$, then every two-coloring of the complete 3-uniform hypergraph on vertices contains a monochromatic copy of the hedgehog $H_{t}$. That is,

$$
r\left(H_{t}\right)<200 t^{2} \ln t+400 t^{2}+1
$$

We make no attempt to optimize the absolute constants here.

\section{Ramsey number of hedgehogs}

Throughout this section, we assume $t \geq 10$, and that we have a fixed two-coloring of the edges of a complete 3-uniform hypergraph $\mathcal{H}$ on vertex set $V$ with $n \geq 200 t^{2} \ln t+400 t^{2}$ vertices. Let

$$
m_{\max }:=2 t+\left(\begin{array}{l}
t \\
2
\end{array}\right)
$$

Let $\left(\begin{array}{l}S \\ 2\end{array}\right)$ denote the set of pairs of elements of $S$. For integer $a$, let $[a]=\{1,2, \ldots, a\}$. For vertices $u$ and $v$ of $\mathcal{H}$, we write $u v$ as an abbreviation for the unordered pair $\{u, v\}$. 
For $u, v \in V$, let

$$
\begin{aligned}
& d_{u v}^{(r)}:=\mid\{w:\{u, v, w\} \text { red }\} \mid \\
& d_{u v}^{(b)}:=\mid\{w:\{u, v, w\} \text { blue }\} \mid .
\end{aligned}
$$

For a set of pairs $F \subset\left(\begin{array}{c}V \\ 2\end{array}\right)$, let

$$
\begin{aligned}
& N^{(b)}(F):=\{w: \exists u v \in F \text { s.t. }\{u, v, w\} \text { blue }\} \\
& N^{(r)}(F):=\{w: \exists u v \in F \text { s.t. }\{u, v, w\} \text { red }\} .
\end{aligned}
$$

Here, and throughout, we use $b$ and $r$ to refer to the colors blue and red, respectively. For a vertex $v$ and set $X$, let

$$
\begin{aligned}
& U_{\leq m}^{(b)}(v, X)=\left\{u \in X: d_{u v}^{(r)} \leq m\right\} \\
& U_{\leq m}^{(r)}(v, X)=\left\{u \in X: d_{u v}^{(b)} \leq m\right\} .
\end{aligned}
$$

If $X$ is omitted, take $X=V$. We define $U_{\leq m}^{(b)}(v, X)$ to be sets of $u$ such that $d_{u v}^{(r)}$ is small, rather than those such that $d_{u v}^{(b)}$ is small, because we wish to think of $U^{(b)}$ 's as sets helpful for finding a blue hedgehog. Similarly, we think of $U^{(r)}$ 's as sets helpful for finding a red hedgehog.

Lemma 2.1. For any $0 \leq m<\frac{|V|}{2}-1$, and $v \in V$,

$$
\min \left(\left|U_{\leq m}^{(b)}(v)\right|,\left|U_{\leq m}^{(r)}(v)\right|\right) \leq 2 m
$$

Proof. Fix $m$ and $v$. For convenience, let $A=U_{\leq m}^{(b)}(v)$ and $B=U_{\leq m}^{(r)}(v)$. Assume for contradiction that $|A|,|B| \geq 2 m+1$. For every $u$, we have $d_{u v}^{(r)}+d_{u v}^{(b)}=|V|-2>2 m$, so $A$ and $B$ are disjoint. Consider the set $E^{\prime}$ of edges of $\mathcal{H}$ containing $v$, one element of $A$, and one element of $B$. On one hand, $\left|E^{\prime}\right|=|A| \cdot|B|$. On the other hand, for every $u \in A$, the pair $u v$ is in at most $m$ such red triples, so the number of red triples of $E^{\prime}$ is at most $|A| \cdot m$. Additionally, for every $u \in B$, the pair $u v$ is in at most $m$ such blue triples, so the number of blue triples of $E^{\prime}$ is at most $|B| \cdot m$. Hence, $(|A|+|B|) \cdot m \leq\left|E^{\prime}\right|=|A| \cdot|B|$, a contradiction of $|A|,|B| \geq 2 m+1$.

The following "matching condition" for hedgehogs is useful.

Lemma 2.2. Let $S \subset V$ be a set of $t$ vertices. If, for all nonempty sets $F \subset\left(\begin{array}{l}S \\ 2\end{array}\right)$, we have $\left|N^{(b)}(F)\right| \geq|F|+t$, then there exists a blue hedgehog with body S. Similarly, if, for all nonempty sets $F \subset\left(\begin{array}{c}S \\ 2\end{array}\right)$, we have $\left|N^{(r)}(F)\right| \geq|F|+t$, then there exists a red hedgehog with body $S$.

Proof. By symmetry, it suffices to prove the first part. Consider the bipartite graph $G$ between pairs in $\left(\begin{array}{l}S \\ 2\end{array}\right)$ and vertices of $V \backslash S$, where $u v \in\left(\begin{array}{l}S \\ 2\end{array}\right)$ is connected with $w \in V \backslash S$ if and only if triple $\{u, v, w\}$ is blue. If, for all nonempty $F \subset\left(\begin{array}{c}S \\ 2\end{array}\right)$, we have $\left|N^{(b)}(F)\right| \geq|F|+t$, then any such $F$ has at least $|F|+t-|S|=|F|$ neighbors in $G$. By Hall's marriage lemma on $G$, there exists a matching in $G$ using every element of $\left(\begin{array}{c}S \\ 2\end{array}\right)$. Taking triples $\{u, v, w\}$ where $u v \in\left(\begin{array}{l}S \\ 2\end{array}\right)$ and $w \in V \backslash S$ is the vertex matched with pair $u v$ gives a blue hedgehog with body $S$. 


\subsection{Special Cases}

We start by finding monochromatic hedgehogs in two specific classes of colorings on $\mathcal{H}$. We base our proof of Theorem 1.2 on the argument for the first class of colorings, which we call simple colorings. We use the result for the second class of colorings, which we call balanced colorings, as a specific case in the general argument.

\subsubsection{Simple colorings}

Consider hypergraphs that are colored the following way:

1. Start with a graph $G$ on $[n]$.

2. Color a complete hypergraph $\mathcal{H}$ on $[n]$ by coloring the triple $\{u, v, w\}$ blue if at least one of $u v, u w, v w$ is in $G$, and red otherwise.

Lemma 2.3. If $n \geq t^{2}+t$, any hypergraph colored as above has a monochromatic $H_{t}$.

Proof. Set $X=V(G)$. For $i=t-1, t-2, \ldots, 0$, pick a vertex $v_{i} \in X$ whose degree in $G$ is at least $i$ and let $\hat{U}\left(v_{i}\right) \subset X$ be an arbitrary set of $i$ neighbors of $v_{i}$. Remove $v_{i} \cup \hat{U}\left(v_{i}\right)$ from $X$. We call this the peeling step of $v_{i}$. Figure 2.1.1 shows the first three peeling steps of this process for $t=5$. If this process succeeds, we have found a set $S=\left\{v_{t-1}, \ldots, v_{0}\right\}$ of $t$ vertices and disjoint sets of vertices $\hat{U}\left(v_{0}\right), \ldots, \hat{U}\left(v_{t-1}\right)$ also disjoint from $S$, from which we can greedily embed a blue-hedgehog in $\mathcal{H}$ with body $\left\{v_{0}, \ldots, v_{t-1}\right\}$ : for each $v_{i} v_{j}$ with $i<j$, pick an arbitrary unused element of $\hat{U}\left(v_{j}\right)$ for the third vertex of the hedgehog's edge containing $v_{i} v_{j}$.

Now suppose this process finds vertices $v_{t-1}, v_{t-2}, \ldots, v_{i+1}$ but fails to find $v_{i}$ for some $i \leq t-1$. After picking $v_{j}$, we remove $v_{j}$ and $j$ of it's neighbors from $X$, for a total of $j+1$ vertices. Then we have removed exactly $t+(t-1)+\cdots+(i+2)=\left(\begin{array}{c}t+1 \\ 2\end{array}\right)-\left(\begin{array}{c}i+2 \\ 2\end{array}\right)$ vertices from $X$. Hence, $|X| \geq\left(t^{2}+t\right)-\left(\begin{array}{c}t+1 \\ 2\end{array}\right)+\left(\begin{array}{c}i+2 \\ 2\end{array}\right)=\left(\begin{array}{c}t+1 \\ 2\end{array}\right)+\left(\begin{array}{c}i+2 \\ 2\end{array}\right)>\frac{t^{2}+i^{2}}{2} \geq t i$, and every vertex has degree at most $i-1$ in the subgraph of $G$ induced $X$. Thus, there exists an independent set $S \subset X$ in $G$ of size at least $|X| / i \geq t$. Furthermore, any vertex has at most $i-1$ neighbors in $X$, so any two vertices $u, v \in S$ share at least $|X|-2 i \geq t+\left(\begin{array}{c}t \\ 2\end{array}\right)+\left(\begin{array}{c}i+2 \\ 2\end{array}\right)-2 i>t+\left(\begin{array}{c}t \\ 2\end{array}\right)$ red triples in the subhypergraph of $\mathcal{H}$ induced by $X$, so we can greedily find a red hedgehog with body $S$.

\subsubsection{Balanced colorings}

In this section, we consider the case where our coloring is "balanced". Lemma 2.1 tells us that, for every vertex $v$ and every nonnegative integer $m$ less than $\frac{|V|}{2}-1$, one of $\left|U_{\leq m}^{(b)}(v)\right|=$ $\#\left\{u: d_{u v}^{(r)} \leq m\right\}$ and $\left|U_{\leq m}^{(r)}(v)\right|=\#\left\{u: d_{u v}^{(b)} \leq m\right\}$ is at most $2 m$. In "balanced" colorings, we assume, for all $v \in V$ and all $2 t \leq m \leq m_{\max }:=2 t+\left(\begin{array}{c}t \\ 2\end{array}\right)$, both of $\left|U_{\leq m}^{(b)}(v)\right|$ and $\left|U_{\leq m}^{(r)}(v)\right|$ are $O(m)$. We show, in this case, there is a monochromatic hedgehog. The proof is by choosing a random subset of approximately $4 t$ vertices, and showing that, with positive probability, we can remove vertices so that the remaining set of $t$ vertices is the body of some red hedgehog. 


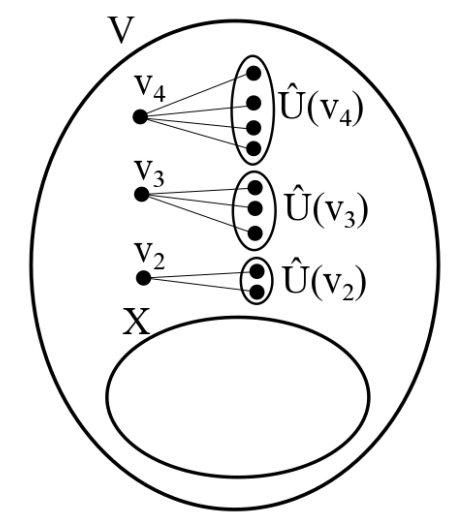

Figure 1: Peeling $v_{4}, v_{3}, v_{2}$ in Lemma 2.3

Lemma 2.4. Let $c \geq 1$. Consider a two-colored hypergraph $\mathcal{H}=(V, E)$ on $n \geq 40 \mathrm{ct}^{2}$ vertices. Suppose that for all $2 t \leq m \leq m_{\max }$ and all $v \in V$, we have

$$
\left|U_{\leq m}^{(b)}(v)\right| \leq c m
$$

Then $\mathcal{H}$ has a red hedgehog $H_{t}$.

Proof. It suffices to prove for $n=40 c t^{2}$, so assume without loss of generality that $n=40 c t^{2}$. Pick a random set $S$ by including each vertex of $V$ in $S$ independently with probability $4 t / n$. By the Chernoff bound, $\operatorname{Pr}[|S| \leq 3 t] \leq e^{-t / 8}$.

Fix $m$ such that $2 t \leq m \leq m_{\max }$ and $m$ is a multiple of $t$. Let $e_{1}, \ldots, e_{p}$ be the pairs such that $d_{e_{\ell}}^{(b)} \leq m$ for all $\ell \in[p]$, and let $X_{1}, \ldots, X_{p}$ the indicator random variables for these pairs being in $\left(\begin{array}{l}S \\ 2\end{array}\right)$. Let $X=X_{1}+\cdots+X_{p}$. By (1), we have $p \leq c m n / 2$. Each $X_{\ell}$ for $\ell \in[p]$ is a Bernoulli $\left(16 t^{2} / n^{2}\right)$ random variable. Consider a graph on $[p]$ where $\ell$ and $\ell^{\prime}$ are adjacent (written $\ell \sim \ell^{\prime}$ ) if $e_{\ell}$ and $e_{\ell^{\prime}}$ share a vertex. This is a valid dependency graph for $\left\{X_{\ell}\right\}$ as $X_{\ell}$ is independent of all $X_{\ell^{\prime}}$ such that $e_{\ell^{\prime}}$ is vertex disjoint from $e_{\ell}$. Furthermore, by the condition (1), each endpoint of any pair $e_{\ell}$ is in at most $\mathrm{cm}$ pairs, so each $\ell \in[p]$ has degree at most $2 \mathrm{~cm}$ in the dependency graph, and the total number of pairs $\left(\ell, \ell^{\prime}\right)$ such that $\ell \sim \ell^{\prime}$ is at most $2 c m p$. We have

$$
\begin{aligned}
\mathbf{E}[X] & =\frac{16 t^{2} p}{n^{2}}=\frac{2 p}{5 c n} \leq \frac{m}{5}<\frac{3 m}{4}-t \\
\operatorname{Var}[X] & =\sum_{\ell, \ell^{\prime} \in[p]} \mathbf{E}\left[X_{\ell} X_{\ell^{\prime}}\right]-\mathbf{E}\left[X_{\ell}\right] \mathbf{E}\left[X_{\ell^{\prime}}\right] \\
& =\sum_{\ell \sim \ell^{\prime}} \mathbf{E}\left[X_{\ell} X_{\ell^{\prime}}\right]-\mathbf{E}\left[X_{\ell}\right] \mathbf{E}\left[X_{\ell^{\prime}}\right] \\
& \leq 2 c m p \cdot\left(\left(\frac{4 t}{n}\right)^{3}-\left(\frac{4 t}{n}\right)^{4}\right) \\
& <\frac{128 t^{3} c m p}{n^{3}} \leq \frac{64 t^{3} c^{2} m^{2}}{n^{2}}=\frac{m^{2}}{25 t}
\end{aligned}
$$


Hence,

$$
\begin{aligned}
\operatorname{Pr}\left[\#\left\{u v \in\left(\begin{array}{c}
S \\
2
\end{array}\right): d_{u v}^{(r)} \leq m\right\}>m-t\right] & =\operatorname{Pr}[X>m-t] \\
& =\operatorname{Pr}[X-\mathbf{E}[X] \geq m-t-\mathbf{E}[X]] \\
& \leq \operatorname{Pr}[X-\mathbf{E}[X] \geq m / 4] \\
& \leq \frac{\operatorname{Var}[X]}{(m / 4)^{2}}<\frac{16}{25 t}
\end{aligned}
$$

The first inequality is by (2) and the second is by Chebyshev's inequality. By the union bound over the multiples of $t$ in $\left[2 t, m_{\max }\right]$, of which there are less than $t$, the probability there exists some $m \in\left[2 t, m_{\max }\right]$ a multiple of $t$ with

$$
\#\left\{u v \in\left(\begin{array}{l}
S \\
2
\end{array}\right): d_{u v}^{(r)} \leq m\right\} \leq m-t
$$

is less than $t \cdot \frac{16}{25 t}=\frac{16}{25}$. Again by the union bound, with probability more than $1-\left(\frac{16}{25}+e^{-t / 8}\right)>$ 0 over the randomness of $S$, we have (i) $|S| \geq 3 t$, and (ii) for all $m$ a multiple of $t$ in $\left[2 t, m_{\max }\right]$, (3) holds. Hence, there exists an $S$ such that (i) and (ii) hold, so consider such an $S$. Remove $|S|-t \geq 2 t$ vertices from $S$, at least one from each of the $2 t$ pairs with smallest $d_{u v}^{(r)}$, to obtain a set of $t$ vertices $T$ such that, for all $m$ a multiple of $t$ in [2t, $\left.m_{\max }\right]$, we have

$$
\#\left\{u v \in\left(\begin{array}{c}
T \\
2
\end{array}\right): d_{u v}^{(r)} \leq m\right\} \leq \max \left(0, \#\left\{u v \in\left(\begin{array}{c}
S \\
2
\end{array}\right): d_{u v}^{(r)} \leq m\right\}-2 t\right) \leq \max (0, m-3 t) .
$$

Then, for all $m$ with $2 t \leq m \leq m_{\max }-t$, set $m^{\prime}$ to be the smallest multiple of $t$ larger than $m$, so that

$$
\#\left\{u v \in\left(\begin{array}{l}
T \\
2
\end{array}\right): d_{u v}^{(r)} \leq m\right\} \leq \#\left\{u v \in\left(\begin{array}{l}
T \\
2
\end{array}\right): d_{u v}^{(r)} \leq m^{\prime}\right\} \leq \max \left(0, m^{\prime}-3 t\right) \leq m-2 t .
$$

Now, we show our matching condition holds. Setting $m=2 t$ in (4), we have $x_{u v}>2 t$ for all $u v \in\left(\begin{array}{l}T \\ 2\end{array}\right)$. Hence, for any nonempty subset $F \subset\left(\begin{array}{l}T \\ 2\end{array}\right)$ of size at most $t$, any $u v \in F$ satisfies $x_{u v}>t+|F|$. If $F \subset\left(\begin{array}{l}T \\ 2\end{array}\right)$ has size greater than $t$, then, by setting $m=t+|F|$ in (4), we know that there are at most $m-2 t=|F|-t$ pairs $u v \in F$ such that $d_{u v}^{(r)} \leq t+|F|$, so again there exists $u v \in F$ such that $d_{u v}^{(r)}>t+|F|$. We conclude that, for all nonempty subsets of pairs $F \subset\left(\begin{array}{c}T \\ 2\end{array}\right)$, there exists $u v \in F$ such that $\left|N^{(r)}(F)\right| \geq d_{u v}^{(r)} \geq t+|F|$. By Lemma 2.2, there exists a red hedgehog with body $T$.

\subsection{Proof of Theorem 1.2}

\subsubsection{Proof outline}

To prove Theorem 1.2, we follow the proof of Lemma 2.3. First, "peel off" vertices $v$ into a set $S$ to try to find a blue or red hedgehog. ${ }^{1}$ If we succeed, we are done. If we fail, we end up with an induced two-colored hypergraph that is "balanced" in the sense of Lemma 2.4. In this case, we simply apply Lemma 2.4 .

\footnotetext{
${ }^{1}$ For technical reasons, we peel vertices to find both blue and red hedgehogs, as opposed to Lemma 2.3 where we only peeled vertices to find a blue hedgehog.
} 

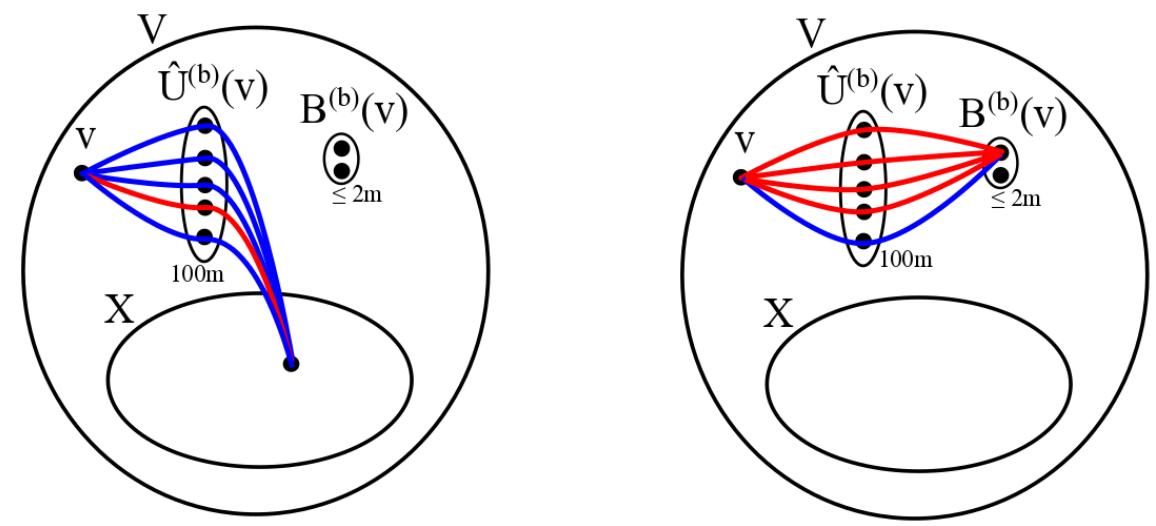

Figure 2: Peeling $v$ with many blue-heavy neighbors. For every $w \in X$, edge $\{u, v, w\}$ is blue for many $u \in \hat{U}^{(b)}(v)$. Vertices $w \in B^{(b)}(v)$ are the exception. Ideally we simply delete vertex $v$, set $\hat{U}^{(b)}(v)$, and set $B^{(b)}(v)$ from $X$ (depicted), but instead we maintain fractional penalties $\alpha^{(\chi)}(\cdot)$ and $\beta^{(\chi)}(\cdot)$. We have $\left|\hat{U}^{(b)}(v)\right|=10 m$ by definition, and $\left|B^{(b)}(v)\right| \leq 2 m$ by Lemma 2.6.

In the proof of Lemma 2.3, we started with $X=V$ and iteratively removed from $X$ a vertex $v$ and a set $\hat{U}(v)$ of size $t$ such that, for all $u \in \hat{U}(v)$, vertices $u$ and $v$ share many blue triples. This deletes $O(t)$ vertices per round, which is small enough for the argument to succeed. For general hypergraphs, we peel off vertices $v$ with many "blue-heavy neighbors", meaning there exists some $m$ such that $\left|U_{\leq m}^{(b)}(v, X)\right| \geq 10 m{ }^{2}$ However, $m$ can be $\Theta\left(t^{2}\right)$, so if we simply deleted $v$ along with $10 m$ of its blue-heavy neighbors $\hat{U}^{(b)}(v) \subset U_{\leq m}^{(b)}(v, X)$, we could delete $\Theta\left(t^{2}\right)$ vertices for every $v$, which is too many. Instead, when we peel off $v$, we delete $v$ from $X$, add a penalty of $t / m$ to each $u \in \hat{U}^{(b)}(v)$, accumulated as $\alpha^{(b)}(u)$, and delete from $X$ every vertex $u$ with $\alpha^{(b)}(u) \geq 1 / 2$. With these penalties, we guarantee that, on average, we delete $O(t)$ vertices from $X$ per peeled vertex $v$.

However, we need more care. In Lemma 2.3, we can find a hedgehog with body $S$ because, for any peeled vertices $v, v^{\prime} \in S$, the edges $\left\{u, v, v^{\prime}\right\}$ are blue for every $u \in \hat{U}(v)$. However, in our procedure, for a $v$ chosen with corresponding $\hat{U}^{(b)}(v)$ of size $10 \mathrm{~m}$, there are some vertices $w$ such that $\{u, v, w\}$ is blue for few (at most $4 m$ ) vertices $u \in \hat{U}^{(b)}(v)$. We denote this set of "bad" vertices by $B^{(b)}(v)$. As much as possible, we wish to avoid choosing both $v$ and, at some later step, $w \in B^{(b)}(v)$ for the body $S^{(b)}$ of our blue hedgehog. Ideally, we simply delete all vertices $u \in B(v)$ in the step we peel off $v$. However, $B^{(b)}(v)$ can have $\Omega(m)$ vertices, which again could be too many if $m=\Theta\left(t^{2}\right)$. Instead, for each $w \in B^{(b)}(v)$ we add a penalty of $t / d_{w v}^{(b)}$, accumulated as $\beta^{(b)}(w)$, and delete from $X$ every vertex $w$ with $\beta^{(b)}(w) \geq 1 / 4$. We guarantee that, on average, we delete $O(t \ln t)$ vertices from $X$ per peeled vertex $v$ (Lemma 2.9).

To finish the proof, we show, if our peeling produces a set $S^{(b)}=\left\{v_{1}, \ldots, v_{t}\right\}$ (where $v_{i}$ is chosen before $\left.v_{i+1}\right)$, then, because we track the penalties $\alpha^{(b)}(u)$ and $\beta^{(b)}(w)$ carefully, the matching condition of Lemma 2.2 holds. On the other hand, if the peeling procedure fails, the subhypergraph induced by $X$ is large and balanced, in which case we apply Lemma 2.4.

\footnotetext{
${ }^{2}$ For technical reasons, we peel vertices $v$ in increasing order of the corresponding $m$.
} 


\subsubsection{The peeling procedure}

We now describe the procedure formally. Start with $S^{(b)}=S^{(r)}=\emptyset$, and $X=V$. For all $u \in V$, initialize $\alpha^{(r)}(u)=\alpha^{(b)}(u)=\beta^{(r)}(u)=\beta^{(b)}(u)=0$. If, at any point, $S^{(b)}$ or $S^{(r)}$ has $t$ vertices, stop.

Recall that $m_{\max }=2 t+\left(\begin{array}{l}t \\ 2\end{array}\right)$. For $m=2 t, 2 t+1, \cdots, m_{\max }$, do the following, which we refer to as $\operatorname{Stage}(m)$.

1. While there exists a vertex $v \in X$ and a color $\chi \in\{b, r\}$ such that $\left|U_{\leq m}^{(\chi)}(v, X)\right| \geq 10 m$ :

(a) Let $\hat{U}^{(\chi)}(v)$ be the set $U_{\leq m}^{(\chi)}(v, X)$ truncated to $10 m$ vertices arbitrarily.

(b) Let $B^{(\chi)}(v)=\left\{w: \mid u \in \hat{U}^{(\chi)}(v):\{u, v, w\}\right.$ is color $\left.\chi \mid \leq 4 m\right\}$.

(c) Add $v$ to $S^{(\chi)}$.

(d) For all $u \in \hat{U}^{(\chi)}(v)$, add $t / m$ to $\alpha^{(\chi)}(u)$.

(e) For all $w \in B^{(\chi)}(v)$, add $\min \left(1 / 4, t / d_{v w}^{(\chi)}\right)$ to $\beta^{(\chi)}(w)$.

(f) Delete from $X$ all vertices $u$ with $\alpha^{(\chi)}(u) \geq 1 / 2$ or $\beta^{(\chi)}(u) \geq 1 / 4$.

(g) Delete $v$ from $X$.

Note that $B^{(\chi)}(v)$ and $\hat{U}^{(\chi)}(v)$ are only defined for $v \in S^{(\chi)}$. We refer to steps $1(\mathrm{a})-1(\mathrm{~g})$ as the peeling step for $v$, denoted $\operatorname{Peel}(v)$. We let $m_{v}$ denote the value such that the peeling step for $v$ occurred during Stage $\left(m_{v}\right)$, and call $m_{v}$ the peeling parameter of $v$. Throughout the analysis, let $X_{v}$ denote the set $X$ immediately before Peel $(v)$. For any $m \in\left[2 t, m_{\max }\right]$, let $X_{m}$ denote the set $X$ immediately after Stage $(m)$, so that $X_{m_{\max }}$ is the set $X$ at the end of the peeling procedure.

The above process terminates in one of two ways. Either we "get stuck", i.e. we complete Stage $\left(m_{\max }\right)$ and $\left|S^{(b)}\right|<t$ and $\left|S^{(r)}\right|<t$, or we "finish", i.e. we terminate earlier with $\left|S^{(b)}\right|=t$ or $\left|S^{(r)}\right|=t$. We show there is a monochromatic hedgehog in each case. In Subsection 2.2.5, we handle the case where we "get stuck". In Subsection 2.2.6, we handle the case where we "finish".

\subsubsection{Basic facts about peeling}

We first establish the following facts about the procedure.

Lemma 2.5. For any $m$ such that $2 t \leq m \leq m_{\max }$, for any time in the procedure after Stage $(m)$, the following holds: for all colors $\chi \in\{b, r\}$, for all $m^{\prime}$ with $2 t \leq m^{\prime} \leq m$, and for all vertices $v \in X$, we have $\left|U_{\leq m^{\prime}}^{(\chi)}(v, X)\right|<10 m^{\prime}$.

Proof. Fix $m$ with $2 t \leq m \leq m_{\max }$. We have $\left|U_{\leq m}^{(\chi)}\left(v, X_{m}\right)\right|<10 m$ for all $v \in X_{m}$ : if not, then there exists a vertex $v \in X_{m}$ with $\left|U_{\leq m}^{(\chi)}\left(v, X_{m}\right)\right| \geq 10 m$, in which case we would have peeled vertex $v$ during Stage $(m)$, and we would have deleted $v$ from $X_{m}$ during $\operatorname{Peel}(v)$, which is a contradiction. Throughout the procedure, $X$ is nonincreasing. Thus, at any point in the procedure after $\operatorname{Stage}(m)$, we have $X \subset X_{m}$, so for all $v \in X$, we have $v \in X_{m}$ and $\left|U_{\leq m}^{(\chi)}(v, X)\right| \leq\left|U_{\leq m}^{(\chi)}\left(v, X_{m}\right)\right|<10 m$. 
Lemma 2.6. For all colors $\chi \in\{b, r\}$ and all vertices $v \in S^{(\chi)}$, we have $\left|B^{(\chi)}(v)\right| \leq 2 m_{v}$.

Proof. We prove this for $\chi=b$, and the case $\chi=r$ follows from symmetry. We doublecount the number $Z$ of red triples $\{u, v, w\}$ such that $u \in \hat{U}^{(b)}(v)$ and $w \in B^{(b)}(v)$. On one hand, every $u \in \hat{U}^{(b)}(v)$ is in at most $m_{v}$ red triples because we chose $\hat{U}^{(b)}(v)$ as a subset of $U_{\leq m_{v}}^{(b)}\left(v, X_{v}\right)$, so the total number of red triples is at most $m_{v} \cdot\left|\hat{U}^{(b)}(v)\right|=10 m_{v}^{2}$. On the other hand, by definition of $B^{(b)}(v)$, each $w \in B^{(b)}(v)$ is in at least $\left|\hat{U}^{(b)}(v)\right|-4 m_{v}=6 m_{v}$ such red triples. Thus, the number of such triples is at least $\left|B^{(b)}(v)\right| \cdot 6 m_{v}$. Hence, $10 m_{v}^{2} \geq$ $Z \geq 6 m_{v}\left|B^{(b)}(v)\right|$ so $\left|B^{(b)}(v)\right| \leq 2 m_{v}$ as desired.

Lemma 2.7. For all colors $\chi \in\{b, r\}$ and all vertices $v, v^{\prime} \in S^{(\chi)}$, we have $d_{v v^{\prime}}^{(\chi)} \geq 4 t$.

Proof. Assume for sake of contradiction that $d_{v v^{\prime}}^{(\chi)}<4 t$. Without loss of generality, $v$ was added to $S^{(\chi)}$ before $v^{\prime}$. We have $d_{v v^{\prime}}^{(\chi)}<4 t<4 m_{v}$, so during Peel $(v)$, vertex $v^{\prime}$ is included in $B^{(\chi)}(v)$. Hence, $\min \left(1 / 4, t / d_{v v^{\prime}}^{(\chi)}\right)=1 / 4$ is added to $\beta_{\leq 4 t}^{(\chi)}\left(v^{\prime}\right)$ during $1(\mathrm{e})$ of $\operatorname{Peel}(v)$, so during $1(\mathrm{f})$ of $\operatorname{Peel}(v)$, vertex $v^{\prime}$ is deleted from $X$ if it hasn't been deleted already. Thus, we could not have added $v^{\prime}$ to $S^{(\chi)}$ after $\operatorname{Peel}(v)$, which is a contradiction, so $d_{v v^{\prime}}^{(\chi)} \geq 4 t$, as desired.

\subsubsection{Bounding the number of deleted vertices}

Lemma 2.8. For all colors $\chi \in\{b, r\}$ and all vertices $v \in S^{(\chi)}$, during $\operatorname{Peel}(v)$, the total increase in $\alpha^{(\chi)}(u)$ over all $u \in V$ is exactly $10 t$.

Proof. Fix $v \in S^{(\chi)}$. We have $\left|\hat{U}^{(\chi)}(v)\right|=10 m_{v}$ by definition, and, for $u \in \hat{U}^{(\chi)}(v)$, each $\alpha^{(\chi)}(u)$ increases by exactly $t / m_{v}$, for a total increase of $10 m_{v} \cdot\left(t / m_{v}\right)=10 t$.

Lemma 2.9. For all colors $\chi \in\{b, r\}$ and all vertices $v \in S^{(\chi)}$, during $\operatorname{Peel}(v)$, the total increase in $\beta^{(\chi)}(w)$ over all $w \in V$ is at most $20 t \ln t$.

Proof. By symmetry, it suffices to prove the lemma for $\chi=b$. Let $v \in S^{(b)}$. For $m=$ $0, \ldots, 4 m_{v}$, let

$$
\begin{aligned}
a_{m} & :=\#\left\{w \in X_{v}: d_{v w}^{(b)}=m\right\} \\
a_{\leq m} & :=a_{0}+a_{1}+\cdots+a_{m}=\left|U_{\leq m}^{(r)}\left(v, X_{v}\right)\right| .
\end{aligned}
$$

$\operatorname{Peel}(v)$ is after Stage $\left(m_{v}-1\right)$. Hence, by Lemma 2.5, for $2 t \leq m \leq m_{v}-1$, we have $a_{\leq m} \leq 10 m$. We know

$$
\left|U_{\leq 4 m_{v}}^{(b)}\left(v, X_{v}\right)\right| \geq\left|U_{\leq m_{v}}^{(b)}\left(v, X_{v}\right)\right| \geq 10 m_{v}>8 m_{v}
$$

where the second inequality holds because $v$ was chosen to be peeled in $\operatorname{Stage}\left(m_{v}\right)$. Hence, by Lemma 2.1, $a_{\leq 4 m_{v}}=\left|U_{\leq 4 m_{v}}^{(r)}\left(v, X_{v}\right)\right| \leq\left|U_{\leq 4 m_{v}}^{(r)}(v)\right| \leq 8 m_{v}$. As $a_{\leq m}$ is non-decreasing in $m$, we conclude $a_{\leq m} \leq 10 m$ for $2 t \leq m \leq 4 m_{v}$.

For $m=0, \ldots, 4 m_{v}$, for any $w$ with $d_{v w}^{(b)}=m$, the peeling of $v$ increases $\beta^{(b)}(w)$ by exactly $\min (1 / 4, t / m)$. Thus, for $a_{m}$ many $w$, the penalty $\beta^{(b)}(w)$ increases by $\min (1 / 4, t / m)$. Furthermore, $\beta^{(b)}(w)$ increases only for $w \in B^{(b)}(v)$, which has at most $2 m_{v}$ vertices by 
Lemma 2.6. For $2 m_{v}-a_{\leq 4 m_{v}}$ vertices $w, \beta^{(b)}(w)$ increases by less than $t / 4 m_{v}$, giving a total increase in $\beta^{(b)}(w)$ of less than $t$ from those vertices. The total increases in $\beta^{(b)}(w)$ is thus less than

$$
\frac{1}{4}\left(a_{0}+a_{1}+\cdots+a_{4 t}\right)+\frac{a_{4 t+1} t}{4 t+1}+\cdots+\frac{a_{4 m_{v}} t}{4 m_{v}}+t
$$

The coefficients of $a_{0}, \ldots, a_{4 m_{v}}$ in (5) are nonincreasing, so (5) is $t$ plus a positive linear combination of $a_{\leq 4 t}, a_{\leq 4 t+1}, \cdots, a_{\leq 4 m_{v}}$. Subject to $a_{\leq m} \leq 10 m$ for $2 t \leq m \leq 4 m_{v}$, all of $a_{\leq 4 t}, a_{\leq 4 t+1}, \ldots, a_{\leq 4 m_{v}}$ are simultaneously maximized when $a_{0}=0$ and $a_{m}=10$ for $m=$ $1, \ldots, 4 m_{v}$, so (5) is maximized there as well. Hence,

$$
\begin{aligned}
\text { Total increase in } \beta^{(b)}(w) & <\frac{1}{4}\left(a_{0}+a_{1}+\cdots+a_{4 t}\right)+\frac{a_{4 t+1} t}{4 t+1}+\cdots+\frac{a_{4 m_{v}} t}{4 m_{v}}+t \\
& \leq t+\frac{1}{4} \cdot 40 t+\frac{10 t}{4 t+1}+\frac{10 t}{4 t+2}+\cdots+\frac{10 t}{4 m_{v}} \\
& \leq 11 t+10 t \ln \left(4 m_{v} / 4 t\right)<20 t \ln t,
\end{aligned}
$$

where, for the last inequality, we used $m_{v} \leq t^{2}$ and $t \geq 10$. This is what we wanted to show.

Lemma 2.10. The total number of vertices deleted from $X$ in the peeling procedure is at most $200 t^{2} \ln t$.

Proof. A vertex is deleted either for being added to $S^{(b)}$ or $S^{(r)}$, having $\alpha^{(b)}(\cdot)$ or $\alpha^{(r)}(\cdot)$ at least $1 / 2$, or having $\beta^{(b)}(\cdot)$ or $\beta^{(r)}(\cdot)$ at least $1 / 4$. At the end of the procedure, we have the following inequalities. For all $\chi \in\{b, r\}$ and all $u \in V$, we have $\alpha^{(\chi)}(u)$ and $b^{(\chi)}(u)$ are initially 0 and increase only during the peeling step of some vertex $v \in S^{(\chi)}$. Hence, by Lemma 2.8, for $\chi \in\{b, r\}$,

$$
\sum_{u \in V} \alpha^{(\chi)}(u)=10 t \cdot\left|S^{(\chi)}\right| \leq 10 t^{2}
$$

Furthermore, by Lemma 2.9, for $\chi \in\{b, r\}$,

$$
\sum_{u \in V} \beta^{(\chi)}(u) \leq 20 t \ln t \cdot\left|S^{(\chi)}\right| \leq 20 t^{2} \ln t .
$$

We conclude that, at the end of the procedure,

$$
\begin{aligned}
\#\{\text { deleted } u\} \leq & \left|S^{(b)}\right|+\left|S^{(r)}\right|+\#\left\{u: \alpha^{(b)}(u) \geq 1 / 2\right\}+\#\left\{u: \alpha^{(r)}(u) \geq 1 / 2\right\} \\
& \quad+\#\left\{u: \beta^{(b)}(u) \geq 1 / 4\right\}+\#\left\{u: \beta^{(r)}(u) \geq 1 / 4\right\} \\
< & 2 t+\sum_{u \in V}\left(2 \alpha^{(b)}(u)+2 \alpha^{(r)}(u)+4 \beta^{(b)}(u)+4 \beta^{(r)}(u)\right) \\
\leq & 2 t+2 \cdot 10 t^{2}+2 \cdot 10 t^{2}+4 \cdot 20 t^{2} \ln t+4 \cdot 20 t^{2} \ln t \\
< & 200 t^{2} \ln t .
\end{aligned}
$$




\subsubsection{Case 1: Peeling procedure gets stuck}

By Lemma 2.10, the number of vertices deleted in the peeling process is at most $200 t^{2} \ln t$, so, at the end of the peeling procedure, $|X| \geq\left(200 t^{2} \ln t+400 t^{2}\right)-200 t^{2} \ln t=400 t^{2}$.

Consider the complete 2-colored subhypergraph $\mathcal{H}^{\prime}$ of $\mathcal{H}$ induced by the vertex set $X$. By Lemma 2.5, at the end of the procedure, for all $m=2 t, 2 t+1, \ldots, m_{\max }$ and all $v \in X$,

$$
\left|U_{\leq m}^{(b)}(v, X)\right|<10 m, \quad\left|U_{\leq m}^{(r)}(v, X)\right|<10 m .
$$

Applying Lemma 2.4 to $\mathcal{H}^{\prime}$ with $c=10$, we conclude $\mathcal{H}^{\prime}$ (and hence $\mathcal{H}$ ) has a red hedgehog $H_{t}{ }^{3}$

\subsubsection{Case 2: Peeling procedure finishes}

Suppose we finish with $\left|S^{(b)}\right|=t$. The analysis for $\left|S^{(r)}\right|=t$ is symmetrical. We try to find a blue hedgehog. For brevity, in the rest of this section, let $S=S^{(b)}$. Let $S=\left\{v_{1}, \cdots, v_{t}\right\}$, where the $v_{i}$ were chosen in the order $v_{1}, \ldots, v_{t}$. For $i=1, \ldots, t$, let $m_{i}=m_{v_{i}}$ be the peeling parameter for $v_{i}$, so that $m_{1} \leq m_{2} \leq \cdots \leq m_{t}$.

Definition 2.11. Call a pair $v_{i} v_{j} \in\left(\begin{array}{c}S \\ 2\end{array}\right)$ with $i<j$ bad if $v_{j} \in B^{(b)}\left(v_{i}\right)$. Otherwise, call $v_{i} v_{j} \in\left(\begin{array}{c}S \\ 2\end{array}\right)$ good. Let $E_{\text {bad }} \subset\left(\begin{array}{c}S \\ 2\end{array}\right)$ be the set of all bad pairs and let $E_{\text {good }} \subset\left(\begin{array}{l}S \\ 2\end{array}\right)$ be the set of all good pairs, so that $\left(\begin{array}{c}S \\ 2\end{array}\right)=E_{b a d} \cup E_{\text {good }}$ is a partition.

\section{Lemma 2.12 .}

$$
\sum_{v_{i} v_{j} \in E_{b a d}} \frac{1}{d_{v_{i} v_{j}}^{(b)}}<\frac{1}{4}
$$

Proof. Fix $2 \leq j \leq t$. Consider all bad pairs $v_{i} v_{j}$ with $i<j$. At the peeling of $v_{j}$, $\beta\left(v_{j}\right)<1 / 4$, otherwise $v_{j}$ would have been deleted from $X$ and we could not have peeled $v_{j}$. Hence, at the peeling of $v_{j}$,

$$
\frac{1}{4}>\beta^{(b)}\left(v_{j}\right)=\sum_{\substack{i: i<j, v_{j} \in B^{(b)}\left(v_{i}\right)}} \min \left(\frac{1}{4}, \frac{t}{d_{v_{i} v_{j}}^{(b)}}\right)=\sum_{\substack{i: i<j, v_{i} v_{j} \in E_{\text {bad }}}} \min \left(\frac{1}{4}, \frac{t}{d_{v_{i} v_{j}}^{(b)}}\right)=\sum_{\substack{i: i<j, v_{i} v_{j} \in E_{\text {bad }}}} \frac{t}{d_{v_{i} v_{j}}^{(b)}}
$$

The first equality is by definition of $\beta^{(b)}\left(v_{j}\right)$, the second is by definition of $E_{b a d}$, and the last is because $d_{v_{i} v_{j}}^{(b)} \geq 4 t$ for all $i<j$ by Lemma 2.7. Thus,

$$
\sum_{v_{i} v_{j} \in E_{\text {bad }}} \frac{1}{d_{v_{i} v_{j}}^{(b)}}=\sum_{j=2}^{t} \sum_{\substack{i: i<j, v_{i} v_{j} \in E_{\text {bad }}}} \frac{1}{d_{v_{i} v_{j}}^{(b)}} \leq \sum_{j=2}^{t} \frac{1}{4 t}<\frac{1}{4}
$$

We prove that there is a blue hedgehog with body $S$, by showing the matching condition of Lemma 2.2 holds. Consider an arbitrary $F \subset\left(\begin{array}{c}S \\ 2\end{array}\right)$. Partition $F=F_{b a d} \cup F_{\text {good }}$, where $F_{b a d}=F \cap E_{b a d}$ and $F_{\text {good }}=F \cap E_{\text {good }}$. We wish to show that $N^{(b)}(F) \geq|F|+t$.

\footnotetext{
${ }^{3} \mathrm{By}$ the same reasoning $\mathcal{H}^{\prime}$ also has a blue hedgehog.
} 
Subcase 1: $\left|F_{\text {bad }}\right| \geq\left|F_{\text {good }}\right|$.

By Lemma 2.12,

$$
\frac{\left|F_{b a d}\right|}{\max _{v_{i} v_{j} \in F_{b a d}} d_{v_{i} v_{j}}^{(b)}} \leq \sum_{v_{i} v_{j} \in F_{b a d}} \frac{1}{d_{v_{i} v_{j}}^{(b)}} \leq \sum_{v_{i} v_{j} \in E_{b a d}} \frac{1}{d_{v_{i} v_{j}}^{(b)}}<\frac{1}{4}
$$

Thus, there exists some $v_{i} v_{j} \in F_{b a d}$ such that $d_{v_{i} v_{j}}^{(b)}>4\left|F_{b a d}\right|$. Furthermore, this $v_{i} v_{j}$ satisfies $d_{v_{i} v_{j}}^{(b)} \geq 4 t$ by Lemma 2.7 , so $d_{v_{i} v_{j}}^{(b)} \geq 2\left|F_{b a d}\right|+2 t$. Hence,

$$
\left|N^{(b)}(F)\right| \geq d_{v_{i} v_{j}}^{(b)} \geq 2\left|F_{b a d}\right|+2 t \geq\left|F_{b a d}\right|+\left|F_{\text {good }}\right|+2 t>|F|+t,
$$

as desired. The first inequality is because the blue edges containing $v_{i} v_{j}$ are all elements of $N^{(b)}(F)$. The second inequality is because $d_{v_{i} v_{j}}^{(b)}$ is at least $4\left|F_{b a d}\right|$ and at least $4 t$ by above. The third inequality is by the assumption $\left|F_{b a d}\right| \geq\left|F_{\text {good }}\right|$. The fourth inequality is because $|F|=\left|F_{\text {bad }}\right|+\left|F_{\text {good }}\right|$ and $2 t>t$.

Subcase 2: $\left|F_{\text {bad }}\right|<\left|F_{\text {good }}\right|$.

In particular, $\left|F_{\text {good }}\right|>0$, so $|F|$ has some good pair $v_{i} v_{j}$ with $i<j$. This pair is in at least $4 m_{i} \geq 8 t$ blue triples, so $\left|N^{(b)}(F)\right| \geq 8 t$.

Let $I$ be the set of all indices $i$ such that there exists $j$ with $i<j \leq t$ with $v_{i} v_{j} \in F_{\text {good }}$. For each $i$, there are less than $t$ indices $j$ such that $i<j \leq t$, so

$$
|I| \cdot t>\left|F_{\text {good }}\right| \cdot
$$

For each $i \in I$, arbitrarily fix $j_{i}>i$ such that $v_{i} v_{j_{i}}$ is good. For $i \in I$, define

$$
U_{i}^{*}:=N^{(b)}\left(\left\{v_{i} v_{j_{i}}\right\}\right) \cap \hat{U}^{(b)}\left(v_{i}\right), \quad U_{I}^{*}:=\bigcup_{i \in I} U_{i}^{*} .
$$

so that $U_{I}^{*} \subset N^{(b)}(F)$. For all $i \in I$, the pair $v_{i} v_{j_{i}}$ is good, so $v_{j_{i}} \notin B^{(b)}\left(v_{i}\right)$. Hence, by the definition of $B^{(b)}\left(v_{i}\right)$, there are more than $4 m_{i}$ vertices $u \in \hat{U}^{(b)}\left(v_{i}\right)$ such that $\left\{u, v_{i}, v_{j_{i}}\right\}$ is blue. Thus, for all $i \in I$, the set $U_{i}^{*}$ has at least $4 m_{i}$ vertices. In the peeling of $v_{i}$, the penalty $\alpha^{(b)}(u)$ increases by $t / m_{i}$ for each $u \in U_{i}^{*}$. Hence, in peeling $v_{i}$, the sum of penalties $\sum_{u \in U_{I}^{*}} \alpha^{(b)}(u)$, increases by at least $4 m_{i} \cdot t / m_{i}=4 t$. Thus,

$$
4 t \cdot|I| \leq \sum_{u \in U_{I}^{*}} \alpha^{(b)}(u) .
$$

On the other hand, the vertex $u$ is deleted from $X$ whenever $\alpha^{(b)}(u) \geq 1 / 2$, the penalty $\alpha^{(b)}(u)$ increases by at most $t / 2 t=1 / 2$ in any peeling step, and the penalty $\alpha^{(b)}(u)$ never changes after $u$ is deleted from $X$. Thus, for all vertices $u \in V$, we have

$$
\alpha^{(b)}(u) \leq 1 .
$$

We conclude

$$
\begin{aligned}
2|F| & \leq 4\left|F_{\text {good }}\right| \leq 4 t|I| \leq \sum_{u \in U_{I}^{*}} \alpha^{(b)}(u) \\
& \leq \sum_{u \in U_{I}^{*}} 1=\left|U_{I}^{*}\right| \leq\left|N^{(b)}\left(F_{\text {good }}\right)\right| \leq\left|N^{(b)}(F)\right| .
\end{aligned}
$$


The first inequality is by the assumption $\left|F_{\text {bad }}\right|<\left|F_{\text {good }}\right|$, the second is by (6), the third is by (7), the fourth is by (8), the fifth is by $U_{I}^{*} \subset N^{(b)}\left(F_{\text {good }}\right)$, and the sixth is by $F_{\text {good }} \subset F$. Combining with $\left|N^{(b)}(F)\right| \geq 8 t$, we conclude $\left|N^{(b)}(F)\right| \geq|F|+t$, as desired.

This covers all subcases, so we've proven that, for any nonempty subset $F \subset\left(\begin{array}{l}S \\ 2\end{array}\right)$, we have $N^{(b)}(F) \geq|F|+t$. Hence, the matching condition of Lemma 2.2 holds, so there is a blue hedgehog with body $S$, as desired. This completes the proof of Theorem 1.2.

\section{References}

[1] N. Alon, M. Krivelevich and B. Sudakov, Turán numbers of bipartite graphs and related Ramsey-type questions, Combin. Probab. Comput. 12 (2003), 477-494.

[2] S. A. Burr and P. Erdős, On the magnitude of generalized Ramsey numbers for graphs, in Infinite and Finite Sets, Vol. 1 (Keszthely, 1973), 214-240, Colloq. Math. Soc. János Bolyai, Vol. 10, North-Holland, Amsterdam, 1975.

[3] D. Conlon, J. Fox and B. Sudakov, Hypergraph Ramsey numbers, J. Amer. Math. Soc. 23 (2010), 247-266.

[4] D. Conlon, J. Fox and B. Sudakov, Recent developments in graph Ramsey theory, Survey in Combinatorics 2015 49-118, London Math. Soc. Lecture Note Ser., 424, Cambridge Univ. Press, Cambridge, 2015.

[5] D. Conlon, J. Fox and V. Rödl, Hedgehogs are not colour blind, J. Comb. 8 (2017), $475-485$.

[6] P. Erdős, A. Hajnal and R. Rado, Partition relations for cardinal numbers, Acta Math. Acad. Sci. Hungar. 16 (1965), 93-196.

[7] P. Erdős and R. Rado, Combinatorial theorems on classifications of subsets of a given set, Proc. London Math. Soc. 3 (1952), 417-439.

[8] J. Fox and B. Sudakov, Two remarks on the Burr-Erdős conjecture, European J. Combin. 30 (2009), 1630-1645.

[9] R. L. Graham, B. L. Rothschild and J. H. Spencer, Ramsey theory, 2nd edition, John Wiley \& Sons, 1990.

[10] A. V. Kostochka and V. Rödl, On Ramsey numbers of uniform hypergraphs with given maximum degree, J. Combin. Theory Ser. A 113 (2006), 1555-1564.

[11] A. V. Kostochka and B. Sudakov, On Ramsey numbers of sparse graphs, Combin. Probab. Comput. 12 (2003), 627-641.

[12] C. Lee, Ramsey numbers of degenerate graphs, Ann. of Math. 185 (2017), 791-829. 